\title{
Denileukin diftitox for the treatment of cutaneous T-cell lymphoma
}

\author{
David Kaminetzky' \\ Kenneth B Hymes ${ }^{2}$ \\ 'Division of Hematology/Oncology, \\ New York University School \\ of Medicine, New York, USA; \\ ${ }^{2}$ New York University Cancer \\ Institute, New York, USA
}

\begin{abstract}
Cutaneous T-cell lymphoma/mycosis fungoides (CTCL/MF) is a rare lymphoproliferative disorder which can present as an indolent or as an aggressive process involving skin, lymph nodes, and blood. In stages IA, IB and IIA, it is usually managed with topical medications and phototherapy. If there is progression despite application of these treatments, or if the patient presents with a higher stage of disease, systemic chemotherapy or retinoids, rexinoids, biologic response modifiers are often necessary. Consequently, patients are often treated with a sequence of modalities and drugs. Denileukin diftitox (DD, Ontak ${ }^{\circledR}$ ) is a targeted immunotoxin which has biological activity against malignancies expressing the IL-2 receptor. In addition to its unique mechanism of action, DD has a toxicity profile which does not overlap with most commonly used chemotherapeutic agents. CTCL/MF has been found be particularly susceptible to treatment with this agent. This review will describe the development DD, its proposed mechanism of action, the clinical trials which identified its utility in the treatment of CTCL/MF, the common toxicities encountered with this agent, and the management of these toxicities. In addition the incorporation of DD in the sequential treatment of CTCL/MF and data suggesting potential combination therapies employing this novel agent will be discussed.
\end{abstract}

Keywords: T-cell lymphoma, mycosis fungoides, immunotoxin, cytokine therapy, denileukin diftitiox

\section{Introduction}

Cutaneous T-cell lymphomas (CTCL) represent less then 3\% of non-Hodgkin's lymphomas (Gemmill 2006). Surveillance Epidemiology and End Results program (SEER) data for CTCL in the US from 1973 to 2002 have shown an overall annual age adjusted incidence of 6.4 per million persons (Criscione and Weinstock 2007). When compared to prior SEER data, this represents a doubling of the incidence, with higher numbers among African Americans and among men. It is seen predominately in the fifth and sixth decades of life (Habermann and Pittelkow 2004).

T-cell lymphomas have been extensively sub-classified (see Table 1) (Willemze et al 2005). Mycosis fungoides represents the largest subgroup; it typically has an indolent course with stereotypical skin eruptions that produce plaques, patches, papules, erythema, alopecia, and/or tumors. The typical skin lesions are often confused with more common diagnoses including psoriasis, fungal infections, and eczema (Hoffman et al 2005). The natural history of this disorder is usually characterized by progressive phases. The initial stages usually consist of flat erythematous patches. Eventually the patches evolve into more infiltrating plaques with discrete borders. These findings are usually asymmetrically distributed and involve areas where there is little exposure to sun. In later stages there is the development of tumor lesions and erythroderma in addition to the plaques and patches (Paulli and Berti 2004). The characteristic histological finding is of epidermal infiltrates with atypical small- to medium-sized T-lymphocytes with cerebriform nuclei. Immunophenotypically, these cells express 
Table I Classification of the common cutaneous lymphomas, associated frequency, and 5-year disease specific survival

\begin{tabular}{lll}
\hline WHO-EORTC classification & Frequency \% & 5-year survival \% \\
\hline Cutaneous T-cell lymphoma & & \\
Mycosis fungoides & 44 & 88 \\
Folliculotropic MF & 4 & 80 \\
PCALCL & 8 & 95 \\
Lymphomatoid papulosis & 12 & 100 \\
Sezary syndrome & 3 & 24 \\
Cutaneous B-cell lymphoma & & \\
PCMZBL & 7 & 99 \\
PCFCL & 11 & 95 \\
PCDLBCL, LT & 4 & 55
\end{tabular}

Adapted from Willemze et al 2005.

Abbreviations: PCALCL, primary cutaneous anaplastic large cell lymphoma; PCMZBL, primary cutaneous marginal zone B-cell lymphoma; PCFCL, primary cutaneous follicular center lymphoma; PCDLBCL, LT, primary cutaneous diffuse large B-cell cell lymphoma, leg type.

CD4+, CD45RO+, and CD3+ markers. A hallmark of the malignant phenotype is the loss of CD5, CD7, CD8, and CD26 antigens. Overall the 5-year survival approaches $88 \%$ (Willemze et al 2005). Sezary syndrome is a more aggressive disease which typically includes the findings of erythroderma, lymphadenopathy, and peripheral circulating lymphoma cells (Rosen and Querfeld 2006). With worsening and more advanced disease states, patients experience increasing pain, pruritus, cutaneous and systemic infections, disfigurement, and visceral involvement. Staging for CTCL is based on the tumor, lymph node, and metastasis (TNM) system. In recent years an additional category for hematological involvement has been incorporated (TNMB) (see Table 2). An overall stage (IA-IVB) can be applied from the TNMB system (Scarisbrick 2006). Prognosis is related to the extent of skin lesions with a higher $\mathrm{T}$ stage corresponding to a reduction in survival (Zackheim et al 1999) as well as to TNM groupings (Kim et al 2003). Standard treatments for localized disease have usually included topical nitrogen mustard, total skin electron beam therapy, and phototherapy. For more advanced disease states interferon, retinoids, extracorporeal phototherapy and systemic chemotherapy have been employed. The results of treatment of advanced CTCL have been disappointing with a high incidence of relapsed disease and treatmentrelated adverse effects. In addition, since the majority of patients with limited disease have relatively good prognosis, any treatment that causes excessive myelosuppression or immunosuppression would be undesirable. Furthermore, it has been proposed that preservation of immune function, particularly CD8+ lymphocytes correlates with improved survival in CTCL (Abeni et al 2005). These observations stress the need for novel agents that can target this disease where traditional treatments have fallen short.

Denileukin diftitox (DD) or DAB389IL-2 (Ontak ${ }^{\circledR}$; Eisai, Inc., Woodcliff Lake, NJ, USA) is a fusion protein in which the receptor binding domain of diphtheria toxin (DT) has been exchanged for that of the interleukin-2 (IL-2) molecule. Because of the specificity of the IL-2 domain, the DT mediated cytotoxic activity will predominately affect cells that express the IL-2 receptor (IL-2R). The IL-2 receptor is selectively expressed on activated T-lymphocytes, B-cells, and natural killer (NK) cells. It has been shown that IL-2 fusion toxin is cytotoxic against in vitro T-cell lines (Williams et al 1987; Kiyokawa et al 1989). It has been estimated that approximately $50 \%$ of CTCL cases express the IL-2R as demonstrated by immunohistochemical staining (Nichols et al 1997).

\section{Mechanism of action}

The IL-2 receptor has been classified into three subtypes based on their affinity for IL-2. Each subtype is composed of a combination of subunits: alpha (CD25, p 55), beta (CD122, p 75), and gamma (CD132, p 64) subunits. The low affinity receptor consists of the IL-2R- $\alpha / \gamma(C D 25$, CD132) subunits that bind to IL-2 but do not cause internalization or activation. In contrast, the intermediate and high affinity receptors that are composed of IL-2R- $\beta / \gamma(\mathrm{CD} 122, \mathrm{CD} 132)$ and Il-2R- $\alpha / \beta / \gamma$ (CD25, CD122, CD133) subunits, respectively, both cause internalization and signal transduction (Re et al 1996). The presence of the $\beta$ and $\gamma(C D 122, C D 132)$ subunits is essential for sensitivity and internalization, respectively, of the fusion toxin. If they are lacking from the tumor cell, DD will be ineffective.

After DD binds to the IL-2 receptor, it is taken up by the cell via receptor mediated endocytosis. Once internalized, acidification occurs; this is vital for the translocation of DT fragment A into the cytoplasm (Bacha et al 1988). The proposed mechanism of action is that the internalized fragment A catalyzes the cleavage of nicotinamide adenine dinucleotide (NAD) to nicotinamide resulting in a transfer of ADP to elongation factor-2 (EF-2). This ADP-ribosylation of EF-2 causes inhibition of mRNA prolongation and ultimately inhibition of protein synthesis.

\section{DAB489-IL-2 clinical trials}

The first form of a DT fusion protein was DAB486-IL-2 which entered clinical trials in the early 1990s. A phase I 
Table 2 TNMB staging system for cutaneous T-cell lymphoma

\begin{tabular}{ll}
\hline Tumor stage & Patches/plaques $<10 \%$ BSA \\
TI & Patches/plaques $>10 \%$ BSA \\
T2 & Tumors \\
T4 & Erythroderma \\
Nodal stage & \\
N0 & No palpable LN, no histological \\
& involvement \\
NI & Palpable LN, no histological \\
& involvement \\
N2 & No palpable LN, + histological \\
& involvement \\
N3 & Palpable LN, + histological \\
Metastatic stage & involvement \\
M0 & \\
MI & No visceral disease \\
Blood stage & Visceral disease \\
B0 & \\
BI & No hematological involvement \\
& Sezary count $>5 \%$ of peripheral \\
blood lymphocytes
\end{tabular}

Adapted from Scarisbrick 2006.

Abbreviations: BSA, body surface area; LN, lymph node.

dose escalation trial in 18 previously treated patients with various hematological malignancies (including 1 patient with CTCL) showed a response rate of approximately $17 \%$, 1 with complete and 2 with partial responses (LeMaistre et al 1992). All the patients in this study had tumors that expressed the p 55 subunit. The maximal treatment dose was limited to $0.1 \mathrm{mg} / \mathrm{kg} /$ day secondary to transient hepatic transaminase elevation. Fever was associated with the administration, which was treated effectively with acetaminophen. No opportunistic infections were observed following this treatment. Prior to receiving the drug, four patients had positive antibodies against DT and two additional patients had evidence of anti-IL-2 antibodies. After treatment many of the patients were found to have antibodies against DAB486-IL-2, DT, and IL-2. Regardless of these findings, an anti-tumor effect was not compromised in the majority of the patients who showed an increase in the titer of these antibodies.

Hesketh and colleagues later explored the role of DAB486-IL-2 in cutaneous T-cell lymphoma in previously treated patients with refractory disease (Hesketh et al 1993). In a limited phase one trial, using 2 dose schedules, 3 out of 5 patients demonstrated a significant response. The range of responses included complete resolution of stage Ia disease for greater than 33 months, partial response in a CTCL/Sezary syndrome patient with marked improvement in skin manifestations and Sezary cell count, and a stage IIa patient who had a partial response with improvement of skin plaques. Overall, both treatment doses were well tolerated. As seen in the study of LeMaistre et al (1993), transient asymptomatic elevation of liver transaminases were noted. Other side effects included mild hypotension, flu-like symptoms, myalgias, fever, chills, and vomiting. All patients had a documented infection during the treatment course (including 1 death); however, it is not clear if this was associated with this medication or represented the natural history of CTCL patients. While all the patients with a significant response had evidence of IL-2R expression, the heterogeneity of this expression was striking and ranged from $40 \%$ to less than $5 \%$ in the analyzed samples. This finding may be related to the limitations of the immunohistochemical assays employed, since this assay only recognized the lowaffinity IL-2R subunit. In addition, the authors point out that in CTCL there may be a background of activated lymphocytes that express IL-2R and may contribute to false positive result. Similar to the findings in the prior study, and contrary to in vitro assays, the presence antibodies against DAB486-IL-2, DT, or IL-2 did not influence the rate of response to treatment.

Prior in vitro observations suggest that cytotoxicity of DAB486-IL-2 is not only dependent on the dose but also on the duration of ligand-receptor interaction. A trial that investigated a longer infusion time of 90 minutes in patients who had prior treatment attempted to address this issue (LeMaistre et al 1993). This trial involved 23 patients including 3 with CTCL. Confirmation of expression of CD 25 (p55) was made in 11 of the 23 patients, with 4 patients being negative and the results for another 8 patients being unknown. While the longer infusions were well tolerated, and achieved serum levels of DAB486-IL-2 which were known to be active in vitro, this treatment schedule failed to show a statistical difference in the area under the concentration curve and the clearance of ligand-toxin complex. These studies of longer infusion times did, however, confirm prior phase one results; clinical responses were seen in a total of 6 patients (4 patients had a reduction of $25 \%$ to $50 \%$ in disease that lasted approximately 4 weeks). All of the responders were CD25 (p55) positive. Of note, 2 of the 3 CTCL patients had a partial and minimal response, with the third having stable disease. A major limitation of this as well of other early studies is the inability to determine if the detection of CD25 represents the presence of high-affinity or low affinity IL-2R.

Another trial involving 20 patients in a dose-escalating 60-minute infusion of DAB486-IL2 showed no significant 
pharmacokinetic difference compared to other trials. Interestingly, 10 patients had CTCL; 1 of these patients with Sezary syndrome had a partial response and 4 others had mixed responses with some skin lesions that resolved while other lesions evolved or remained stable (Kuzel et al 1993). As in the prior studies, DT, or IL-2 antibody titers of the subjects were measured both pre- and post-treatment. While this study did not draw any specific conclusions about the effect of these antibodies, the authors suggested that a less immunogenic fusion peptide might be subject to less antibody-mediated neutralization.

A phase II study involving 14 patients with CTCL again proved the clinical efficacy of this drug (Foss et al 1994). In this report, 1 of 14 patients had a partial response and 2 had a major biologic response (defined as improvement in skin lesions but without clearance of neoplastic cells from the peripheral blood). As in the other studies, the presence of anti DT antibodies did not preclude response to treatment, and presence of CD25 did not differentiate between responders and non-responders.

\section{DAB389-IL-2 clinical trials}

Modifications of DAB486-IL-2 with an internal in-frame deletion of 97 amino acids from the receptor binding domain produced a fusion protein with a 5-fold greater affinity for target cells. This modification also yielded an approximately 10-fold increase in the potency of the molecule, and an overall increase in its half life (Williams et al 1990; Lemaistre et al 1998). This new fusion toxin was named DAB389-IL-2 and later became known as DD.

Saleh and colleagues initiated the first phase I/II clinical trial evaluating the efficacy of this new formulation included 35 patients with CTCL. Of this group, there was a $34 \%$ response rate over various dose ranges with 5 complete and 7 partial responses (Saleh et al 1998). In addition, many of the patients reported significant improvement in their symptoms. Common side effects that were encountered included fever and chills, transient elevation in hepatic transaminases, and nausea.

In a subsequent smaller phase II trial, 4 heavily pretreated patients with CTCL received 9 or $18 \mu \mathrm{g} / \mathrm{kg}$ DAB389IL-2 daily for 5 days followed another course 21 days later for a total of 8 cycles (Duvic et al 1998). These patients had skin plaques which involved the mean of $36 \%$ of the body surface area. Three of the patients received the $18 \mu \mathrm{g} / \mathrm{kg}$ dose and 1 patient received the $9 \mu \mathrm{g} / \mathrm{kg}$ dose. One patient achieved a complete pathological response, another patient had a complete clinical response, and the 2 remaining patients had partial responses. The duration of response ranged from 5 to greater than 11 months, and the majority of reduction in disease burden was noted after the second cycle. As in previous trials, there was no relationship noted between the presence of IL-2R $\alpha$ and clinical response. Importantly, patients reported marked improvement in symptoms and quality of life with some patients returning to work.

In a larger phase I dose-escalating trial, 73 patients with various hematological malignancies were enrolled to receive up to 8 courses of DAB389-IL-2 given as a 5-minute infusion for 5 days with additional treatments every 21 days. In order to be enrolled in this trial, all tumors required expression, via immunohistochemical assay, of either the CD25 or CD122 subunits of IL-2 receptor. Of the 35 patients enrolled with CTCL, 30 had mycosis fungoides, 4 patients had large-cell CTCL, and 1 patient had a peripheral T-cell lymphoma. The stages of disease ranged from stage IA to stage IVB, with the majority of patients being heavily pretreated. Of the CTCL patients, 5 patients had complete and 8 patients had partial responses (overall RR 37\%; 95\% CI 21\%-53\%) (LeMaistre et al 1998; Saleh et al 1998). The median time to response was 2 months with a median duration of response of approximately 10 months. Dose-limiting toxicity was seen at $31 \mu \mathrm{g} / \mathrm{kg} /$ day dose for 5 days and included asthenia, fever, nausea, and vomiting as the predominant symptoms. Unlike DAB486-IL-2, changes in liver function test were not dose limiting. Interestingly, some of the patients with CTCL were found to have edema, hypotension, and hypoalbuminemia, suggestive of a capillary leak-syndrome. As seen with the DAB486-IL-2 studies, the presence of antibodies to DT or DAB389IL-2 did not prevent a treatment response. Of note, there was no correlation between the expressions of either of the subunits and a clinical response. An association may have been seen if CD132 was assayed as this has subsequently been shown to be necessary for a response (Re et al 1996).

The pivotal phase III trial, a randomized, blinded, multicenter study to determine the efficacy, pharmacokinetics, and toxicity of 2-dose regimens of DD in patients who received prior treatment for stage IB to IVA CTCL, was reported by Olsen and colleagues. In order to be included, participants had to have greater than $20 \%$ of lymphocytes within the skin biopsy site stain positive for CD25. Patients were randomly assigned to receive either a 9 or $18 \mu \mathrm{g} / \mathrm{kg} /$ day dose for 5 consecutive days; treatment was repeated every 21 days for up to 8 cycles. There was further randomization of the population into those with $\leq$ IIA and $\geq$ IIB disease. At the conclusion of the study, 71 patients were enrolled; 
$68 \%$ of these patients had stage IIB or more advanced disease. The study yielded an approximately $30 \%$ response rate (CI, 18\%-41\%) with $10 \%$ of patients exhibiting a complete response. The overall response rate between the 2 dosage groups did not reach statistical difference; however there was a trend to superiority in efficacy of the higher dose in patients with greater than stage IIB disease. The median duration of response for those with an objective result was almost seven months with a range from 2.7 to greater than 46 months. A quality of life questionnaire showed a significant difference in symptoms of the respondents with an improvement in the patients' global skin assessment, generalized erythroderma, and pruritus. Approximately $68 \%$ of patients had a significant clinical improvement of their pruritus. In addition, the use of rescue medications (topical steroids and oral anti-histamines) was decreased in those patients who responded. Importantly, $32 \%$ of the patients who did not qualify as having an objective response had stable disease. Of these almost half had a 50\% reduction of their lesions that lasted less than 6 weeks and, therefore, could not be qualified as a partial response (Olsen et al 2001).

As in the prior studies using DAB486-IL-2 and DAB389IL-2, the presence of anti-DT and anti-DD antibodies were measured both at baseline and after each subsequent course. While a relatively minor proportion of patients had detectable levels of both anti-DT and anti-DD antibodies before treatment, in almost all patients these titers became positive and/or increased after treatment. There was no evidence that this rise in antibody titer impaired the response to treatment. Pharmacokinetic data seem to show more rapid clearance of DD by course 3 , which may be related to significant elevation in anti-DD antibodies in the majority of patients at around the same time. Additional data seem to suggest that this is probably a function of the individual antibody that is produced in each patient since no linear relationship was noted. Interestingly, elevated titers of anti-DD were actually associated with less elevation in liver transaminases, hypoalbuminemia, and development of a rash.

\section{Toxicities and management}

Adverse effects including hypersensitivity-like reactions occurred in the majority of patients especially after the first infusion. In the study, $85 \%$ of patients complained of post-treatment flu-like symptoms of mild to moderate severity. Other common adverse events included fever, chills, asthenia, arthralgia, headache, and myalgia, all of which appeared to be managed well with antipyretics.
Gastrointestinal symptoms were also frequently seen. Various forms of a rash were also noted of mild to moderate severity that usually responded well to oral antihistamines and oral/topical steroids. Lymphopenia, the most common hematologic abnormality, was seen in approximately $70 \%$ of the patients. This abnormality was transient with a return to baseline levels usually within 2 weeks. While many patients suffered infectious complications, no causal relationship to treatment was identified. It is important to note, premedication with steroids and anti-histamines were not permitted with this clinical trial. Consequently, the observed toxicities were expected to be more severe than those experienced by patients receiving effective premedication.

Again observed was the presence of a vascular-leak syndrome which the authors characterized by at least 2 of the following: anisarca, hypoalbuminemia, and hypotension. Based on this definition, it may have occurred in up to $25 \%$ of the patients. This number may be somewhat inflated because the majority of the hypotensive events could have been attributed to hypersensitivity reactions rather than true vascular-leak syndrome. The vascular-leak syndrome was usually seen in the first 14 days after receiving treatment and the majority of the patients who were re-treated did not have a recurrence. It was typically self-limiting and treatment with diuretics resulted in improvement. There was a slightly higher incidence of hypotension and hypoalbuminemia in the $18 \mu \mathrm{g} / \mathrm{kg} /$ day dose group.

Considering the risk of vascular-leak syndrome with the lack of clear superior efficacy with the higher dose, the authors suggested that the dose should be determined on a case- by-case basis. With these results, the US Food and Drug Administration approved the use of DD in patients with who have relapsed CTCL and are CD25 positive.

The significant risk of both infusion related hypersensitivity reaction and the vascular leak syndrome was addressed in a small study of 15 patients with CTCL who received either dexamethasone or prednisone premedication (Foss et al 2001). The incidence of acute hypersensitivity reaction and vascular leak syndrome was seen in 3 and 2 patients, respectively, all without compromising clinical response. There are also some limited data to suggest that steroid pre-medication may induce expression of CD25 on T-cells and this correlated with increased response rate (Duvic 2006). Denileukin diftitox can be administered in the outpatient setting via a peripheral line. Pre-medication protocols usually consist of dexamethasone $4 \mathrm{mg}$ iv, diphenyhydramine 25 to $50 \mathrm{mg}$ iv, and acetaminophen $650 \mathrm{mg}$ po (Dang et al 2007). Following infusion, adequate saline hydration should 
continue since this has also shown to diminish the incidence of vascular leak syndrome (Duvic 2006).

Since receiving FDA approval there have been case reports of thyrotoxicosis related to DD administration (Ghori et al 2006). Eight patients with mycosis fungoides receiving either 9 or $18 \mu \mathrm{g} / \mathrm{kg} /$ day for 5 days every 3 weeks developed tremors, tachycardia, nausea, diarrhea, and weight loss. Clinical hyperthyroidism usually developed by the time of the second cycle of treatment. Many of these symptoms were initially related to side effects from this medication. Discontinuation of DD caused resolution of the thyrotoxicosis. Five of the patients later developed long-standing hypothyroidism requiring thyroid replacement therapy. There have also been some limited case reports of DD associated retinopathy; however, the true incidence of this event or its mechanism have not been rigorously studied (Ruddle et al 2006).

\section{Combination therapies}

With clear treatment efficacy exhibited by DD in patients with CTCL, many groups explored ways to improve on its treatment effects. Shao and colleagues addressed modification of tumor cells so that they can express high and intermediate affinity IL-2 receptors and therefore have a greater cytotoxic effect after DD exposure (Shao et al 2002). Arginine butyrate, a histone deacetylase (HDAC) inhibitor, was shown to increase IL-2R $\beta$ gene expression and resulting p 75 (CD122) presence. Cells exposed to this compound and DD showed a clear increase in cell death.

This observation of in vitro synergy between DD is of particular interest since vorinostat, another HDAC inhibitor, has been approved by the FDA for the treatment of CTCL. This finding provides a rationale for combination therapy with DD and vorinostat or other HDAC inhibitors in the treatment of CTCL.

Gorgun and Foss investigated the role of the rexinoids bexarotene (Targretin ${ }^{\circledR}$ ) and alitretinoin $\left(\right.$ Panretin $^{\circledR}$ ) as modulators of high affinity IL-2R expression (Gorgun and Foss 2002). Bexarotene has been used as a standard treatment for patients with early and advanced stage CTCL. These compounds are ligands that bind to members of the retinoic acid receptor family including RAR, RXR, PPAR, and VDR. Bexarotene binds to the retinoic X receptor (RXR) while alitretinoin binds to the retinoic acid receptor and RXR. Once bound, transcription of genes involved in cell growth, differentiation, and apoptosis can occur. Rexinoids have immunomodulatory function and have been shown to increase IL-2 receptor expression on T-cells. This study showed that both bexarotene and alitretinoin increased the expression of CD25 (p 55) and CD75 (p 75) in CTCL cell line. Cells that were subsequently exposed to DD showed a $50 \%$ to $70 \%$ decrease in protein synthesis. This observation suggests that increases in expression of components of the IL-2R can be achieved by retinoids and rexinoids, and that may enhance the cytotoxicity of DD.

A phase I trial investigating the use of bexarotene in conjunction with DD in patients with relapsed or refractory CTCL was undertaken. In this dose-escalating trial bexarotene at doses of 75 to $300 \mathrm{mg} /$ day was combined with DD at a dose of $18 \mu \mathrm{g} / \mathrm{kg} /$ day $\times 3$ every 21 days. Of the 12 evaluable patients, there was a $67 \%$ response rate, 4 patients exhibiting a complete response and another 4 showing a partial response (Foss et al 2005). CD25 expression on circulating CD4+ cells after bexarotene treatment was compared with baseline levels. Of the 12 patients, 8 showed greater than $50 \%$ increase in CD25 expression. This included all 4 patients with a complete response and 1 patient with a partial response. The effect on the IL-2R expression occurred at a relatively low dose of bexarotene of $150 \mathrm{mg} /$ day. There was also some evidence of increased CD25 expression by a normal population of CD4+ cells. Although bexarotene increased expression of $\mathrm{CD} 25$, the authors concluded that because a relatively small sample size no definitive relationship could be made between an objective clinical response and upregulation of CD25 expression. In addition, bexarotene may act as a potent immunomodulator and possibly play a role in the cytotoxic effect of this combination, although not via this proposed mechanism. Hypertriglyceridemia and hypothyroidism were seen, which was attributed to bexarotene and occurred at a similar incidence as in prior studies of this medication. The incidence of grade 3 or 4 lymphopenia was seen in $57 \%$ of the all patients enrolled; this rate is considerably higher than that seen in prior trials of either bexarotene or DD as single agents. Although suggestive of a synergistic effect, this relationship is not clear, since the majority of the patients at baseline had some element of lymphopenia. Regardless as to the cause, this abnormality resolved shortly after discontinuation of the medications, and there were no adverse effects attributed to opportunistic infections.

Recently, Negro-Vilar and colleagues presented a meta-analysis analyzing the efficacy and safety of DD in 3 phase III clinical trials (Negro-Vilar et al 2008). The data indicate that both the 9 and $18 \mu \mathrm{g} / \mathrm{kg}$ iv daily dose regimen for 5 days, every 28 days, showed a significant overall response rate, progression-free survival, and progression of disease when compared to placebo. In addition, there 
was no correlation between CD25 expression and response. Adverse events were generally mild to moderate with a few more severe events seen in the treatment group. The toxicity of treatment resolved to placebo levels after the second or third course of treatment.

\section{Conclusion}

Some 20 years have elapsed since the initial work on a diphtheria fusion toxin. In that time, clinical trials of DD in CTCL have shown significant efficacy against this disease. This has correlated to better outcomes and overall improvement in quality of life for many patients. The clinical trials cited in this paper demonstrate that DD has a measurable response rate of $\sim 30 \%$ to $40 \%$ in patients with CTCL, and that these responses are seen in heavily pre-treated patients with all stages of this disease. In addition, in some patients the duration of the response is prolonged. However, several clinical and laboratory observations have proven to be impediments to a wider use of this compound. First, the identification of the subset patients with CTCL who benefit from this agent is difficult, as the biologic markers which might predict response (CD25 expression) or non-response (presence of anti DT or IL-2 antibodies) do not correlate with the clinical outcomes. Second, the concern over treatmentrelated toxicity and the currently recommended dose schedule make treatment with drug unattractive, particularly to physicians who have limited experience in the treatment of CTCL. Finally, the introduction of bexarotene and vorinostat, both oral agents with similar response rates and durations of response to DD, has shifted the intravenous drug to a lower position in the hierarchy of CTCL treatments.

These difficulties, however, provide an opportunity for additional investigation in the use of DD. First, the development of more reliable reagents which permit the accurate identification of the high, low and intermediate affinity IL-2R will allow for the sub-classification of patients with CTCL (or other IL-2R expressing malignancies). Second, a more general appreciation of the pre-medication regimens which reduce infusion-related toxicity and the vascular leak syndrome should encourage the wider use of DD in CTCL. Finally, the availability of 3 distinct classes of medications (rexinoids, DD, and HDAC inhibitors) with unique mechanisms of actions and non-overlapping toxicities provides many opportunities to investigate combinations of these drugs and to identify more tolerable and effective therapy.

\section{Disclosures}

The authors have no conflicts of interest to disclose.

\section{References}

Abeni D, Frontani M, Sampogna F, et al. 2005. Circulating CD8+ lymphocytes, white blood cells, and survival in patients with mycosis fungoides. Br J Dermatol, 153:324-30.

Bacha P, Williams DP, Waters C, et al. 1988. Interleukin 2 receptor-targeted cytotoxicity. Interleukin 2 receptor-mediated action of a diphtheria toxin-related interleukin 2 fusion protein. J Exp Med, 167:612-22.

Criscione VD, Weinstock MA. 2007. Incidence of cutaneous T-cell lymphoma in the United States, 1973-2002. Arch Dermatol, 143:854-59.

Dang NH, Fayad L, McLaughlin P, et al. 2007. Phase II trial of the combination of denileukin diftitox and rituximab for relapsed/refractory B-cell non-Hodgkin lymphoma. Br J Hematol, 138:502-5.

Duvic M, Cather J, Maize J, et al. 1998. DAB389IL2 diphtheria fusion toxin produces clinical responses in tumor stage cutaneous T-cell lymphoma. Am J Hematol, 58:87-90.

Duvic M. 2006. Optimizing denileukin diftitox (Ontak $\left.{ }^{\circledR}\right)$ therapy. Haematologica Reports, 3:57-60.

Foss FM, Borkowski TA, Gilliom M, et al. 1994. Chimeric fusion protein toxin DAB486-IL-2 in advanced mycosis fungoides and the Sezary syndrome: correlation of activity and interleukin-2 receptor expression in a phase II study. Blood, 84:1765-74.

Foss FM, Bacha P, Osann KE, et al. 2001. Biological correlates of acute hypersensitivity events with DAB389IL-2 (denileukin diftitox, ONTAK) in cutaneous T-cell lymphoma: decreased frequency and severity with steroid premedication. Clin Lymphoma Myeloma, 1:298-302.

Foss F, Demierre MF, Divenuti G. 2005. A phase-1 trial of bexarotene and denileukin diftitox in patients with relapse or refractory cutaneous T-cell lymphoma. Blood, 106:454-7.

Foss F. 2006. Clinical experience with denileukin diftitox. Semin Oncol, 33:S11-6.

Gaffen SL, Liu KD. 2004. Overview of interleukin-2 function, production, and clinical applications. Cytokine, 28:109-23.

Gemmill R. 2006. Cutaneous T-cell lymphoma. Semin Oncol Nurs, 22:90-6.

Ghori F, Polder KD, Pinter-Brown LC, et al. 2006. Thyrotoxicosis after Denileukin Diftitox Therapy in Patients with Mycosis Fungoides. $J$ Clin Endocrinol Metab, 91:2205-8.

Gorgun G, Foss F. 2002. Immunomodulatory effects of RXR retinoids: modulation of high-affinity IL-2R expression enhances susceptibility to denileukin diftitox. Blood, 100: 1399-403.

Habermann TM, Pittelkow MR. 2004. Cutaneous T-cell lymphoma. In Abeloff (ed). Clinical oncology, 3rd ed. Churchill Livingstone. Chapter 113.

Hesketh P, Caguicoa P, Kah H, et al. 1993 Clinical activity of a cytotoxic fusion protein in the treatment of cutaneous T-cell lymphoma. J Clin Oncol, 11:1682-90.

Hoffman R, Benz EJ, Shattil SJ, et al. 2005. Cutaneous T-cell lymphoma. Hematology: basic principles and practice, 4th ed. Philadelphia: Elsevier. Chapter 81.

Kim, YH, Liu HL, Mraz-Gernhard S, et al. 2003. Long-term outcome of 525 patients with mycosis fungoides and Sézary syndrome: clinical prognostic factors and risk for disease progression. Arch Dermatol, 139:857-66.

Kiyokawa T, Shirono K, Hattori T, et al. 1989. Cytotoxicity of Interleukin-2 toxin toward lymphocytes from patients with adult T-cell leukemia. Cancer Res, 49:4042-6.

Kuzel TM, Rosen ST, Gordon LI, et al. 1993. Phase I trial of the diphtheria toxin/interleukin-2 fusion protein DAB486IL-2: efficacy in mycosis fungoides and other non-Hodgkin's lymphoma. Leuk Lymphoma, 11:369-77.

LeMaistre CF, Meneghetti C, Rosenblum, J, et al. 1992. Phase 1 trial of interleukin-2 (IL-2) fusion toxin (DAB486-IL-2) in hematologic malignancies expressing the IL-2 receptor. Blood, 10:2547-54.

LeMaistre CF, Craig FE, Meneghetti C, et al. 1993. Phase I trial of a 90-minute infusion of the fusion toxin DAB486IL-2 in hematological cancers. Cancer Res, 53:3930-4. 
LeMaistre CF, Saleh MN, Kuzel TM, et al. 1998. Phase I trial of a ligand fusion-protein (DAB389IL-2) in lymphomas expressing the receptor for interleukin-2. Blood, 91:399-405.

Negro-Vilar A, Prince HM, Duvic M et al. 2008. Efficacy and safety of denileukin diftitox in cutaneous T-cell lymphoma patients: integrated analysis of three large phase III trials. J Clin Oncol, 26 (May 20 Suppl): Abstract 8551.

Nichols J, Foss F, Kuzel TM, et al. 1997. Interleukin-2 fusion protein: an investigational therapy for interleukin-2 receptor expressing malignancies. Eur J Cancer, 33:534-6.

Olsen E, Duvic M, Frankel A, et al. 2001. Pivotal phase III trial of two dose levels of denileukin diftitox for the treatment of cutaneous T-cell lymphoma. J Clin Oncol, 19:376-88.

Paulli M, Berti E. 2004. Cutaneous T-cell lymphomas (including rare subtypes). Current concepts II. Haematologica, 89:1372-88.

Re GG, Waters C, Poisson L, et. al. 1996. IL-2 receptor expression and sensitivity to diphtheria fusion toxin DAB389-IL-2 in cultured hematopoietic cells. Cancer Res, 56:2590-5.

Rosen ST, Querfeld C. 2006. Primary cutaneous T-cell lymphoma. In Berliner N, Linker C, Schiffer C. (eds) ASH Educational Book 2006. Washington D.C.: American Society of Hematology. p. $323-30$.
Ruddle JB, Harper CA, Höneman D, et al. 2006. A denileukin diftitox (Ontak) associated retinopathy? Br J Ophthalmol, 90:1070-1.

Saleh MN, LeMaistre CF, Kuzel TM, et al. 1998. Antitumor activity of DAB389IL-2 fusion toxin in mycosis fungoides. $J$ Am Acad Dermatol, 39:63-73.

Scarisbrick JJ. 2006. Staging and management of cutaneous T-cell lymphoma. Clin Exp Dermatol, 31:181-6.

Shao RH, Tian X, Gorgun G, et al. 2002. Arginine butyrate increases the cytotoxicity of DAB389IL-2 in leukemia and lymphoma cells by upregulation of IL-2R $\beta$ gene. Leuk Res, 26:1077-83.

Willemze R, Jaffe ES, Burg G, et al. 2005. WHO-ERCT classification for cutaneous lymphoma. Blood, 105:3768-85.

Williams DP, Parker K, Bacha P, et al. 1987. Diphtheria toxin receptor binding domain substitution with Interleukin-2: genetic construction and properties of a diphtheria toxin-related interleukin-2 fusion protein. Protein Eng, 1:493-8.

Williams DP, Snider CE, Strom TB, et al. 1990. Structure function analysis of IL-2 toxin (DAB486-IL-2): Fragment B sequences required for delivery of fragment A to the cytosol of target cells. J Biol Chem, 265:11885-9.

Zackheim, H, Amin, S, Kashani, M, et al. 1999. Prognosis in cutaneous T-cell lymphoma by skin stage: Long-term survival in 489 patients. $J$ Am Acad Dermatol, 40:418-25. 